Article

\title{
Calcium-Free and Cytochalasin B Treatment Inhibits Blastomere Fusion in 2-Cell Stage Embryos for the Generation of Floxed Mice via Sequential Electroporation
}

\author{
Takuro Horii, Ryosuke Kobayashi, Mika Kimura, Sumiyo Morita and Izuho Hatada * \\ Laboratory of Genome Science, Biosignal Genome Resource Center, Institute for Molecular and Cellular \\ Regulation, Gunma University, 3-39-15 Showa-machi, Maebashi 371-8512, Japan; horii@gunma-u.ac.jp (T.H.); \\ rkobayashi@gunma-u.ac.jp (R.K.); mikimura@gunma-u.ac.jp (M.K.); msumiyo@gunma-u.ac.jp (S.M.) \\ * Correspondence: hatada@gunma-u.ac.jp; Tel.: +81-27-220-8057
}

Received: 27 March 2020; Accepted: 27 April 2020; Published: 28 April 2020

\begin{abstract}
The generation of conditional knockout mice using the Cre-loxP system is advantageous for the functional analysis of genes. Flanked by two loxP sites (floxed) mice can be directly obtained from fertilized eggs by the CRISPR/Cas9 genome editing system. We previously reported that sequential knock-in (KI) of each loxP site by electroporation (EP) at the 1- and 2-cell embryonic stages increases the number of mice with floxed alleles compared with simultaneous KI. However, EP at the 2-cell stage frequently induced blastomere fusion. These fused embryos cannot develop to term because they are tetraploidized. In this study, we examined the following three conditions to inhibit blastomere fusion by EP at the 2-cell stage: (1) hypertonic treatment, (2) Calcium $\left(\mathrm{Ca}^{2+}\right)$-free treatment, and (3) actin polymerization inhibition. Hypertonic treatment of 2-cell stage embryos prevented blastomere fusion and facilitated blastocyst development; however, $\mathrm{KI}$ efficiency was decreased. $\mathrm{Ca}^{2+}$-free treatment and actin polymerization inhibition by cytochalasin $\mathrm{B}(\mathrm{CB})$ reduced fusion rate, and did not have negative effects on development and KI efficiency. These results suggest that $\mathrm{Ca}^{2+}$-free and $\mathrm{CB}$ treatment at the 2-cell stage is effective to generate floxed mice in combination with a sequential EP method.
\end{abstract}

Keywords: CRISPR/Cas9; knock-in; flox; electroporation; 2-cell; fusion

\section{Introduction}

Various genome engineering technologies have been developed to establish gene knockout mice; however, a considerable number lead to embryonic lethal phenotypes [1]. The conditional knockout system allows for spatial and temporal control of genetic modification that can overcome this issue. The Cre-loxP conditional knockout system is the most commonly used. The Cre-loxP method utilizes site-specific Cre recombinase and the loxP site consisting of a unique 34-bp sequence [2]. Cre-mediated recombination occurs by deletion or inversion of a genetic region of interest, flanked by two loxP sites (floxed), leading to targeted gene modification in cells expressing Cre.

Until recently, the generation of floxed mice was a time-demanding, complicated process, involving homologous recombination in embryonic stem cells (ESCs), chimeric mouse production by microinjection of these ESCs, and the production of heterozygous offspring by crossing chimeric mice with wild-type mice. Advances in genome editing tools, such as the direct injection of engineered endonucleases or RNA-guided nucleases into zygotes, has greatly accelerated the production of gene-modified animals. The most popular system, clustered regularly interspaced short palindromic repeats (CRISPR)/CRISPR-associated 9 (Cas9), is based on RNA-guided nucleases [3]. The CRISPR/Cas9 
technology enables the generation of knockout mice by the delivery of Cas9 nuclease and guide RNA (gRNA) to target complementary DNA sequences. Furthermore, co-injection of single- or double-stranded donor DNA templates, homologous to target sequences flanking the double strand break (DSB) site, can facilitate precise point mutations or DNA insertions [4-6]. Notably, floxed alleles can be generated by the simultaneous injection of Cas9, two pairs of gRNAs and two single-stranded oligodeoxynucleotides (ssODNs) containing loxP sequences into mouse zygotes [6-9]. This powerful method simplifies the process by eliminating the need to construct a knock-in (KI) vector; thus, floxed mice can be obtained in a short period of time. However, the method using two pairs of gRNAs and ssODNs causes undesirable chromosomal deletion by inducing two DSBs on the same chromosome, therefore reducing the floxed rate $[10,11]$.

To solve this, we developed a method to sequentially introduce each loxP site into the locus of interest at the 1- and 2-cell embryonic stages, respectively (Figure 1) [10]. Sequential electroporation (EP) improved the floxing efficiency compared with ordinary simultaneous microinjection, leading to a high yield of offspring with floxed alleles. However, EP at the 2-cell stage frequently induced blastomere fusion, resulting in tetraploid embryos. Tetraploid embryos arrest development a few days after implantation due to their poor formation of epiblast [12]. Therefore, it is necessary to reduce the rate of blastomere fusion at the 2-cell stage to increase the number of viable embryos. In this study, we examined the following three potential conditions to inhibit blastomere fusion by EP at the 2-cell stage: (1) hypertonic treatment, (2) $\mathrm{Ca}^{2+}$-free treatment, and (3) actin polymerization inhibition. This study shows that $\mathrm{Ca}^{2+}$-free treatment and inhibition of actin polymerization at the 2-cell stage are effective to reduce blastomere fusion, and maintain embryonic development and KI efficiency.

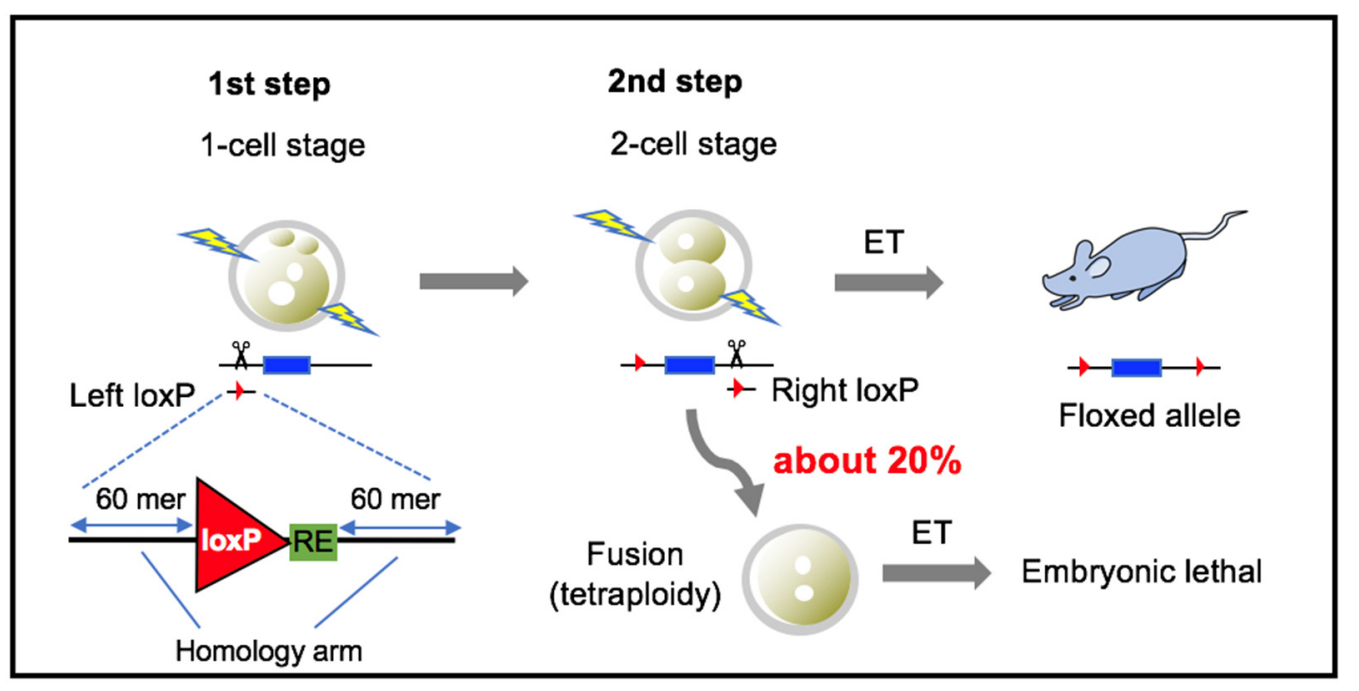

Figure 1. Schematic of sequential EP procedure. At the 1-cell stage, one loxP sequence is inserted by EP. At the 2-cell stage, the other loxP sequence is inserted by EP. Blastomere fusion occurred in $20 \%$, reducing the number of available embryos. $\mathrm{RE}=$ restriction enzyme recognition site, $\mathrm{ET}=$ embryo transfer.

\section{Materials and Methods}

\subsection{Mouse}

B6D2F1 mice were purchased from CLEA Japan (Kawasaki, Japan). C57BL/6J (B6) and ICR mice were purchased from Charles River Japan (Yokohama, Japan). The mice were maintained on a $12 \mathrm{~h}$ light/dark cycle (lights on from 08:00 $\mathrm{h}$ to 20:00 h) and were provided with food and water ad libitum. All animal experiments were approved by the Animal Care and Experimentation Committee of Gunma University (No. 17-030), and were carried out in accordance with the approved guidelines. 


\subsection{Preparation of Embryos}

B6D2F1 (8-10 weeks of age) or C57BL/6J (4 weeks of age) female mice were induced to superovulate by injecting 7.5 units of pregnant mare's serum gonadotropin (PMSG; SEROTROPIN, ASKA Pharmaceutical, Tokyo, Japan) at approximately 16:00 h, followed by the administration of 7.5 units of human chorionic gonadotropin (hCG; GONATROPIN, ASKA Pharmaceutical) $48 \mathrm{~h}$ later. After the administration of the hCG, females were mated with male mice of the same strain. Zygotes were isolated from the oviduct $21 \mathrm{~h}$ later. After washing in M2 medium (Sigma-Aldrich, St. Louis, MO) including $0.1 \%(w / v)$ bovine hyaluronidase, zygotes were transferred to M16 medium (Sigma-Aldrich) supplemented with penicillin and streptomycin at $37^{\circ} \mathrm{C}$. For the in vitro culture of B6 embryos, M16 medium containing $1 \mathrm{mM}$ EDTA/2Na was used to reverse developmental arrest at the 2-cell stage. EP at the 1-cell stage was conducted at 24-26 h post hCG; EP at the 2-cell stage was conducted at $42-44 \mathrm{~h}$ post hCG.

\subsection{Preparation of Cas $9, c r R N A$, tracrRNA and $s s O D N$}

Two CRISPR RNAs (crRNAs) and ssODNs with 5' - and 3'-homology arms (each 60 mer) flanking loxP variants (lox66 or lox71) were designed to target Mecp 2 intron 2 and 3, based on our previous report (Table 1) [10]. To facilitate the detection of correct insertions, the ssODNs were engineered to contain an NheI restrictionsite and an EcoRI restriction site, respectively, in addition to the loxP sequences. Equal volumes of crRNA (100 $\mu \mathrm{M}$; IDT, Coralville, IA) and trans-activating crRNA (tracrRNA) $\left(100 \mu \mathrm{M}\right.$; IDT) were combined in a duplex buffer (IDT), heated in a thermal cycler to $95^{\circ} \mathrm{C}$ for $5 \mathrm{~min}$, and then placed for $10 \mathrm{~min}$ at room temperature according to the manufacture's protocol. Combined crRNA/tracrRNA $(3 \mu \mathrm{M})$ was mixed with recombinant Cas9 protein $(100 \mathrm{ng} / \mu \mathrm{L}$; GeneArt Platinum ${ }^{\mathrm{TM}}$ Cas 9 Nuclease, Thermo Fisher Scientific, Waltham, MA, USA) and ssODNs (400 ng/ $\left.\mu \mathrm{L}\right)$ in Opti-MEM I (Life Technologies, Carlsbad, CA, USA), and the mixture was placed for $10 \mathrm{~min}$ at room temperature before EP.

Table 1. crRNA and ssODN used in this study.

\begin{tabular}{|c|c|c|}
\hline Locus & crRNA & ssODN \\
\hline $\begin{array}{l}\text { Mecp } 2 \text { intron } 2 \\
\text { (Left) }\end{array}$ & $\begin{array}{c}\text { Mecp2-L2 } \\
\left(5^{\prime} \text {-CCCAAGGATACAGTATCCTA-3') }\right.\end{array}$ & $\begin{array}{c}\text { Mecp2-L2-lox66 } \\
\text { (5'-ccagcaacctaaagctgttaagaaatctttgggccccagcttga } \\
\text { cccaaggatacagtatgctagcTACCGTTCGTATAA } \\
\text { TGTATGCTATACGAAGTTATCCTAGGga } \\
\text { agttaccaaaatcagagatagtatgcagcagccaggggtctcat } \\
\text { gtgtggca-3') }\end{array}$ \\
\hline $\begin{array}{l}\text { Mecp2 intron } 3 \\
\quad \text { (Right) }\end{array}$ & $\begin{array}{c}\text { Mecp2-R1 } \\
\text { (5'-AGGAGTGAGGTCTAGTACTT-3') }\end{array}$ & $\begin{array}{c}\text { Mecp2-R1-lox71 } \\
\text { (5'-ccactcctctgtactcctggcttttccacaatccttaaactgaagg } \\
\text { agtgaggtctagtTACCGTTCGTATAGCATACA } \\
\text { TTATACGAAGTTATGAATTCacttgggggtcatt } \\
\text { gggctagactgaata } \\
\text { tctttggttggtacccagacctaatccacca-3') }\end{array}$ \\
\hline
\end{tabular}

Homology arms (lowercase), Restriction enzyme recognition sites (underlined).

\subsection{Electroporation}

EP was performed as described previously [10]. In brief, the electrode (LF501PT1-10; BEX, Tokyo, Japan) connected with the CUY21EDIT electroporator (BEX) was set under a stereoscopic microscope. Embryos were washed twice with Opti-MEM I solution and placed in a line in the electrode gap filled with $5 \mu \mathrm{L}$ Cas9/crRNA/tracrRNA/ssODN mixture. Electroporation was performed using $30 \mathrm{~V}$ (3 msec $\mathrm{ON}+97 \mathrm{msec} \mathrm{OFF}$ ) with seven electric pulses. To determine in vitro development and KI efficiency, embryos were cultured in M16 medium at $37^{\circ} \mathrm{C}$ until the blastocyst stage. To obtain newborn mice, 2-cell stage embryos were transferred to the oviduct of pseudopregnant ICR females. 


\subsection{Inhibition of Blastomere Fusion}

For hypertonic treatment, a $\mathrm{NaCl}$ solution was added to the EP mixture instead of Opti-MEM I. For example, adjusting to $+0.1 \mathrm{M}, 1 \mu \mathrm{L} 1 \mathrm{M} \mathrm{NaCl}$ solution was added to $9 \mu \mathrm{L}$ Opti-MEM I based isotonic EP buffer. $\mathrm{NaCl}$ molarity $(w / v)$ was adjusted to $+1 \mathrm{M}(+5.8 \%),+0.5 \mathrm{M}(+2.9 \%),+0.3 \mathrm{M}(+1.8 \%)$, $+0.2 \mathrm{M}(+1.2 \%)$ and $+0.1 \mathrm{M}(+0.6 \%)$, compared with the normal EP mixture. For cadherin inhibition, 2-cell embryos were treated with $\mathrm{Ca}^{2+}$ and $\mathrm{Mg}^{2+}$-free PBS (PBS (-)) or $\mathrm{Ca}^{2+}$-free M2 medium [13] for $20 \mathrm{~min}$ at $37^{\circ} \mathrm{C}$ before (and after) EP. For actin polymerization inhibition, 2-cell stage embryos were cultured in M2 medium containing $5 \mu \mathrm{g} / \mathrm{mL}$ cytochalasin B (CB, Sigma-Aldrich) at $37^{\circ} \mathrm{C}$ for $20 \mathrm{~min}$ before and/or after EP. After the treatment, embryos were returned to M16 medium and incubated at $37^{\circ} \mathrm{C}$. The number of fused embryos were counted $1 \mathrm{~h}$ after incubation in M16 medium.

\subsection{Assay for KI and Floxed Allele}

Genomic DNA of blastocysts and newborn mice was extracted using DirectPCR Lysis Reagent (Viagen Biotech, Los Angeles, CA, USA) according to the manufacture's protocol. To detect loxP insertion in the target Mecp2 gene, the following primers were used: for intron 2 (Left PCR), Mecp2loxPCheck-1 (5'-AAGAAGCCAACCATACAGTGC-3') and Mecp2loxPCheck-3 (5'-TGAGTGCCACACATGAGACC-3'); for intron 3 (Right PCR), Mecp2loxPCheck-4 (5'-GGGTAGGAAGGCTAGGATGG-3') and Mecp2loxPCheck-2 (5'- GCTTGCTCAGAAGCCAAAAC-3'); for the floxed allele insertion (Long PCR), Mecp2loxPCheck-1 (5'-AAGAAGCCAACCATACAGTGC-3') and Mecp2loxPCheck-4 (5'- GGGTAGGAAGGCTAGGATGG-3'). The expected sizes of the PCR products of the wild-type allele for the Left, Right, and Long PCR were 238, 276, and $983 \mathrm{bp}$, respectively.

PCR was performed in a $10 \mu \mathrm{L}$ reaction volume. The PCR conditions were as follows: initial denaturation $\left(94^{\circ} \mathrm{C}\right.$ for $1 \mathrm{~min}$ ); followed by 40 cycles (for blastocysts) or 35 cycles (for newborn mice) for denaturation $\left(94{ }^{\circ} \mathrm{C}\right.$ for $\left.10 \mathrm{~s}\right)$, annealing $\left(60^{\circ} \mathrm{C}\right.$ for $\left.30 \mathrm{~s}\right)$, and polymerization $\left(72{ }^{\circ} \mathrm{C}\right.$ for $1 \mathrm{~min}$ ); and a final extension at $72{ }^{\circ} \mathrm{C}$ for $5 \mathrm{~min}$ using rTaq DNA polymerase (TaKaRa Taq; \#R001A, Takara Shuzo, Shiga, Japan). To detect KI efficiency, PCR products were digested with NheI and/or EcoRI enzymes, which cleave inserted alleles including loxP sites. The PCR products and digested samples were resolved on a $2 \%$ agarose gel and visualized by staining with ethidium bromide. Alternatively, samples were analyzed using capillary and microchip electrophoresis (MCE-202 MultiNA; Shimadzu, Kyoto, Japan).

\subsection{Statistical Analysis}

The diameter of the blastomeres and the radius $(r)$ of the adhesive area were measured using ImageJ software $(\mathrm{NIH})$. The adhesive area $\left(\mu \mathrm{m}^{2}\right)$ between blastomeres was calculated using a following formula: $3.14 \times r^{2}$. Student's $t$-test (two-tailed test) was applied to calculate $p$ values for them. Fisher's exact probability test was used to calculate $p$ values when comparing fusion, burst, development, born and KI efficiency. A $p$-value of $<0.05$ was considered significant.

\section{Results}

\subsection{Experiment 1: Hypertonic Treatment}

Sequential EP is generally performed under isotonic conditions. Previous reports, aimed at hybridoma production, demonstrated that EP performed under hypotonic conditions increases the rate of cell fusion [14,15]. This effect could be attributed to the improved physical contact between adjacent cells, or the enhanced fusogenic state of the cell membrane. We hypothesize that EP inhibits cell fusion when performed under hypertonic conditions (Figure 2A). We examined five hypertonic conditions $(+1 \mathrm{M},+0.5 \mathrm{M},+0.3 \mathrm{M},+0.2 \mathrm{M}$, and $+0.1 \mathrm{M} \mathrm{NaCl})$ by the addition of a $\mathrm{NaCl}$ buffer to the EP mixture. Under all hypertonic conditions, the blastomeres were observed to shrink, and the cytoplasm became dense and concentrated (Figure 2B and Figure S1A). The blastomeres reduced in size depending on the tonicity of the EP buffer (Figure 2C). Preliminary experiments of EP at the 
2-cell stage (2-cell EP) were performed with a constant voltage ( $30 \mathrm{~V})$. In this condition, about $20 \%$ of 2-cell stage embryos were fused by electric stimulation. As hypothesized, hypertonic conditions containing a minimum of $+0.3 \mathrm{M} \mathrm{NaCl}$ inhibited electric fusion between two blastomeres (Figure 2D). By contrast, the most hypertonic condition $(+1 \mathrm{M} \mathrm{NaCl})$ reduced blastocyst formation (Figure 2D and Figure S1B). The current value under the constant voltage condition is positively correlated with the concentration of $\mathrm{NaCl}$, which is added to the EP buffer. The $+1 \mathrm{M} \mathrm{NaCl}$ condition increased the electric current compared with the control $(0.61 \mathrm{~A}$ vs. $0.17 \mathrm{~A})$, causing damage and reduced embryonic development. To examine EP under constant current $(0.20 \mathrm{~A})$, the voltage was altered. Under constant current and hypertonic conditions, electric fusion was completely inhibited in all hypertonic conditions (Figure 2E) and blastocyst formation was significantly improved compared with the control (Figure 2E and Figure S1C).

Next, the effects of hypertonic treatment were tested with a sequential EP procedure. The first EP was performed under isotonic conditions at the 1-cell stage, followed by a second EP at the 2-cell stage under hypertonic $(+0.1 \mathrm{M} \mathrm{NaCl})$ or normal isotonic conditions. Under hypertonic conditions, blastomere fusion was inhibited, facilitating blastocyst formation (Figure 2F). However, KI efficiency was significantly decreased in the second EP step, indicating a decreased floxed rate compared with control conditions (Figure 2G). Thus, sequential EP combined with hypertonic treatment is unsuitable for efficient generation of floxed mice.

\subsection{Experiment 2: $\mathrm{Ca}^{2+}$-Free Treatment}

Cadherins are a family of cell adhesion receptors that are crucial for the mutual association of vertebrate cells [16]. E-cadherin is essential for the development of preimplanted mouse embryos. E-cadherin null embryos show severe developmental abnormalities at the morula and blastocyst stages [17-19]. In particular, the adhesive cells of the morula dissociate shortly after compaction has occurred, destroying their morphological polarization. E-cadherin null embryos at the 2-cell stage show normal morphology; however, individual blastomeres dissociate after the removal of the zona pellucida [19]. Therefore, temporary inhibition of E-cadherin in electroporated 2-cell stage embryos could repress blastomere adhesion and lead to a reduced fusion rate (Figure 3A). The cadherins are transmembrane glycoproteins that play a key role in $\mathrm{Ca}^{2+}$-dependent cell-cell adhesion, and the 2-cell stage embryo loses blastomere adhesion in the absence of $\mathrm{Ca}^{2+}$ [20]. According to this report, 5-45 min of $\mathrm{Ca}^{2+}$-free treatment is sufficient for the loss of blastomere adhesion. Based on this, 2-cell stage embryos were treated with $\mathrm{Ca}^{2+}$-free PBS (PBS(-)) for $20 \mathrm{~min}$. After a treatment of PBS(-), the two blastomeres were observed to dissociate (Figure $3 \mathrm{~B}$ ) and the adhesive area between blastomeres was significantly reduced (Figure $3 \mathrm{C}$ ). All EP experiments were conducted with the 2-cell stage embryo (2-cell EP). PBS(-) treatment was applied either before EP (-/+) or spanning EP (-/-) (Figure 3D). Almost all embryos (-/+) were viable, whereas $48 \%$ of embryos (-/-) burst during PBS (-) treatment after EP and were not viable (Figure S2A,B). The blastomere fusion rate was significantly reduced by the PBS(-) treatment (Figure S2A,B), but the developmental rate of surviving diploid embryos declined (Figure S2A,C). Therefore, PBS(-) treatment is unsuitable for inhibiting fusion in 2-cell stage embryos.

Next, we examined $\mathrm{Ca}^{2+}$-free $\mathrm{M} 2$ embryo culture medium, with more enriched medium composition than PBS(-), to inhibit blastomere fusion. As expected, $\mathrm{Ca}^{2+}$-free M2 treatment also inhibited blastomere fusion following EP (Figure 3E,F). The fusion rate of blastomeres was $22 \%$ in control embryos, compared with $12 \%$ in embryos $(-/+)$ and $11 \%$ in embryos $(-/-)$. This result indicates that $\mathrm{Ca}^{2+}$-free treatment before EP is necessary to inhibit blastomere fusion. Fortunately, embryos treated with $\mathrm{Ca}^{2+}$-free M2 did not show a reduction in viability, development and KI rates (Figure 3E). To investigate the effects of $\mathrm{Ca}^{2+}$-free treatment on flox efficiency, sequential EP was performed. The first EP was performed at the 1-cell stage under standard conditions; the second EP at the 2-cell stage was performed under $\mathrm{Ca}^{2+}$-free or standard conditions. It was observed that $\mathrm{Ca}^{2+}$-free treatment before EP significantly inhibited cell fusion at the 2-cell stage (Figure 3G). Importantly, $\mathrm{Ca}^{2+}$-free treatment did not have negative effects on blastocyst development and flox efficiency after sequential EP (Figure 3G,H). 
A

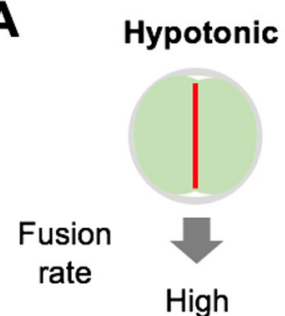

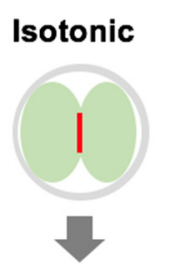

About 20\%
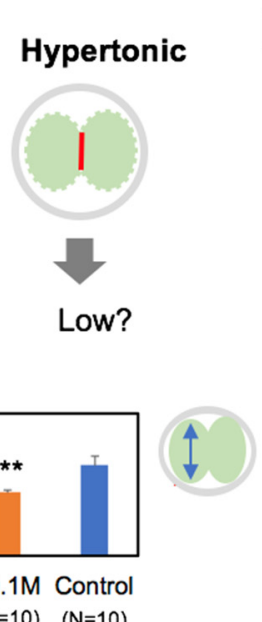

B

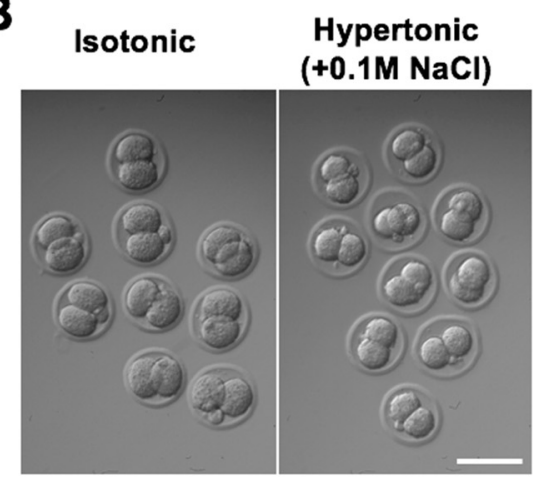

C

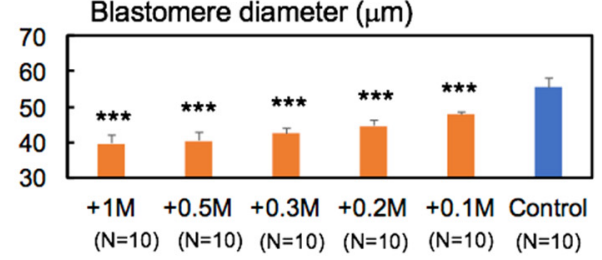

\section{2-cell EP}

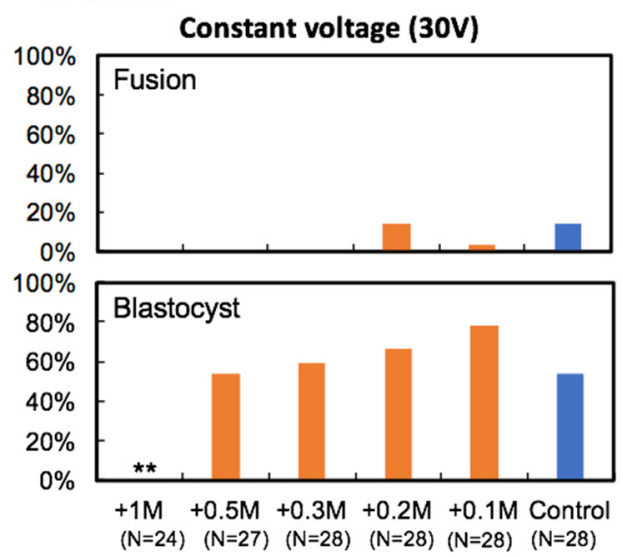

E

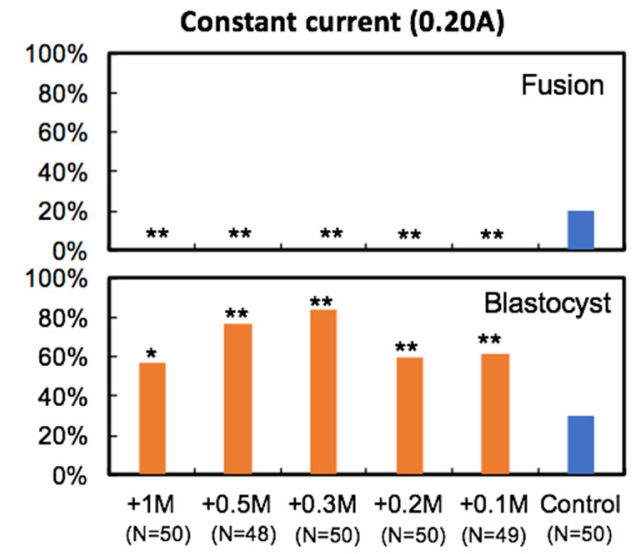

F Sequential EP

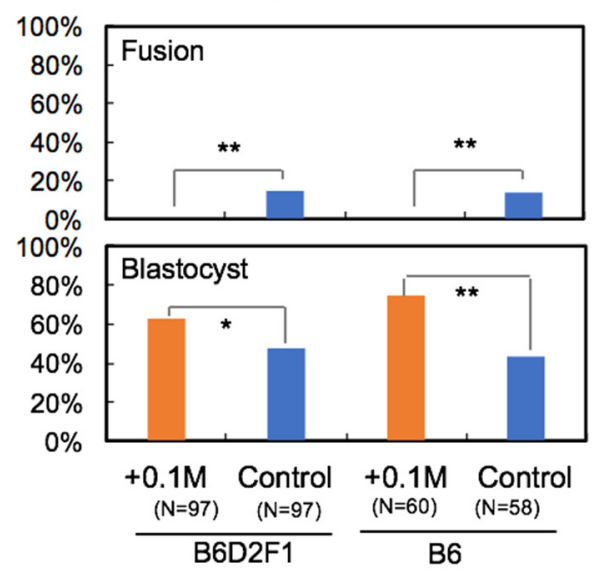

G

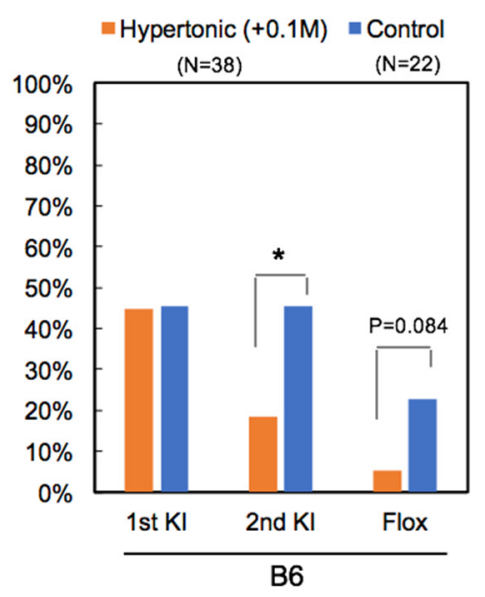

Figure 2. Effects of hypertonic treatment on blastomere fusion, embryonic development and KI efficiency of 2-cell stage embryos. (A) Expected relationship between tonicity and cell fusion. (B) Morphology of 2-cell stage embryos (B6D2F1) after 20 min treatment of isotonic or hypertonic EP buffer. (C) Blastomere diameter under hypertonic conditions. (D) Fusion and development (blastocyst) rate of 2-cell EP embryos under constant voltage. (E) EP under constant current. (F) Fusion and development (blastocyst) rate of 2-cell stage embryos after sequential EP under constant current. (G) KI efficiency of blastocysts by sequential EP under hypertonic or isotonic conditions. $\mathrm{N}$ indicates the number of 2-cell stage embryos (D-F) and blastocysts (G) used for the analysis. ${ }^{*} p<0.05,{ }^{* *} p<0.01,{ }^{* * *} p<0.001$ (compared with control). Scale bar, $100 \mu \mathrm{m}$. 
A
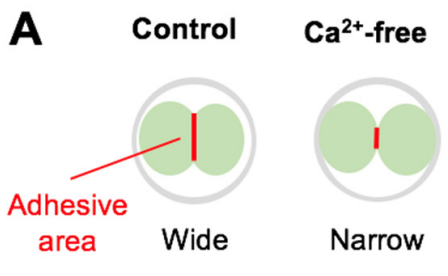

C

Adhesive area

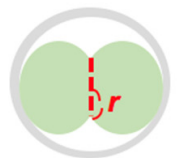

$3.14 \times r^{2}$

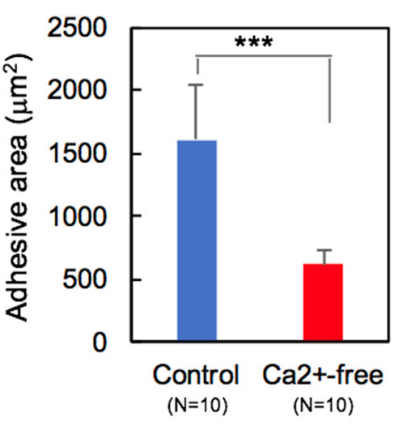

F

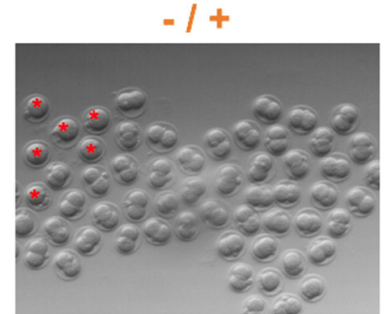

\section{G}

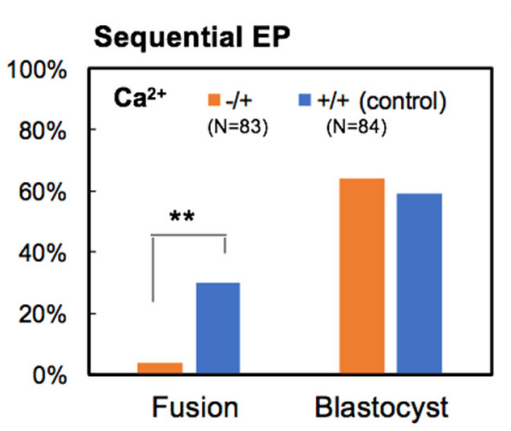

D

(control)

\section{E}

B
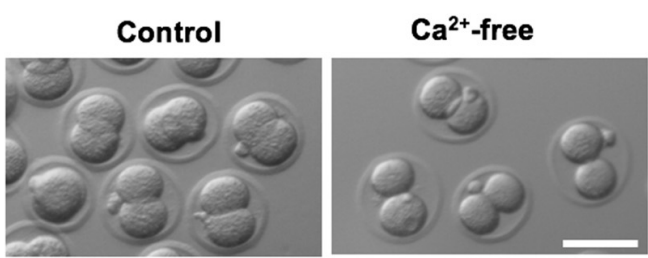
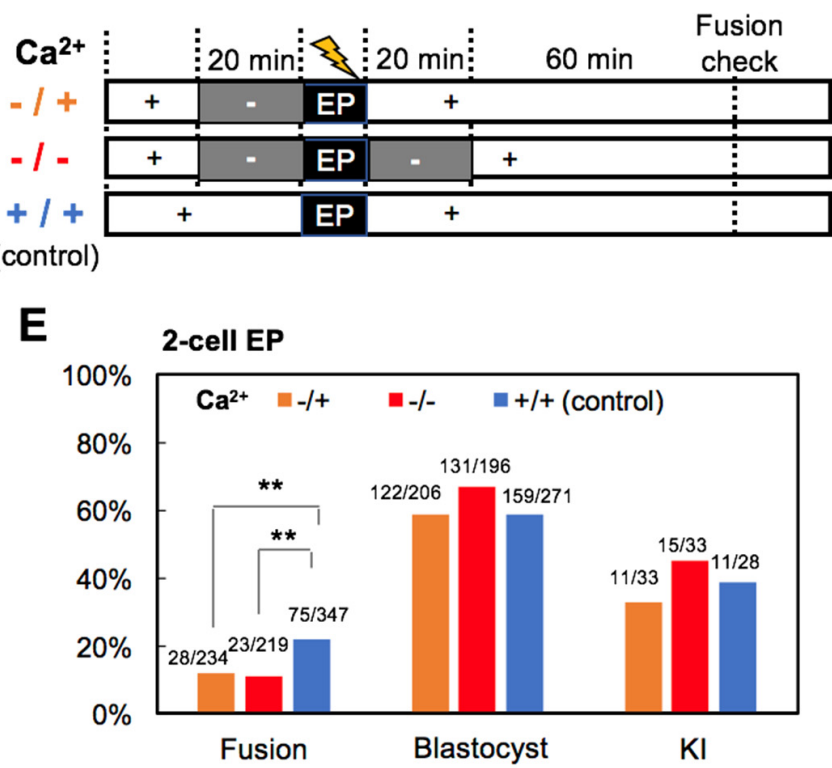

$-1-$

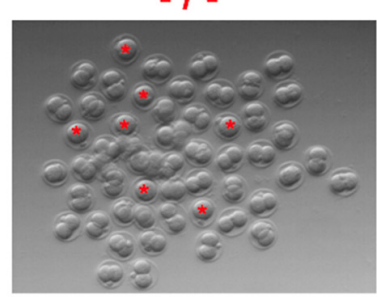

$+/+($ control $)$

H

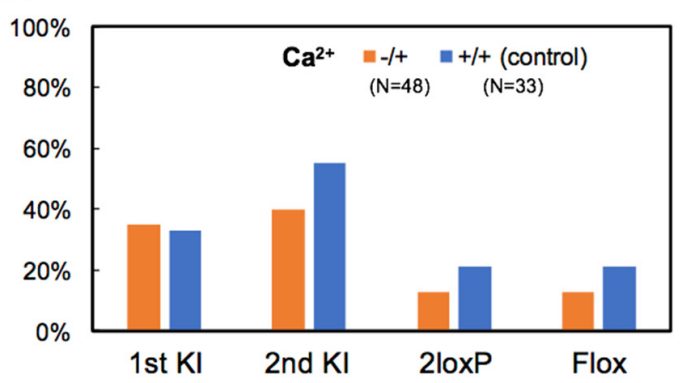

Figure 3. Effects of $\mathrm{Ca}^{2+}$-free buffer treatment on blastomere fusion and embryonic development of 2-cell EP. (A) Relationship between adhesive area and Cadherin inhibition by $\mathrm{Ca}^{2+}$-free treatment. (B) Morphology of 2-cell stage embryos (B6D2F1) after 20 min treatment of $\mathrm{Ca}^{2+}$-free buffer (PBS). (C) Adhesive area beween blastomeres. (D) Experimental timeline of $\mathrm{Ca}^{2+}$-free treatment. (E) Fusion, development (blastocyst) and KI rate by 2-cell EP after $\mathrm{Ca}^{2+}$-free M2 treatment. (F) Morphology of 2-cell EP embryos. Asterisks indicate fused embryos. (G) Fusion and development (blastocyst) rate of 2-cell stage embryos after sequential EP. (H) KI efficiency of blastocysts by sequential EP. 2loxP = embryos with two loxP sites in cis or trans; flox = emryos with two loxP sites in cis. $\mathrm{N}$ indicates the number of 2-cell stage embryos (G) and blastocysts $(\mathbf{H})$ used for the analysis. ${ }^{* *} p<0.01,{ }^{* * *} p<0.001$ (compared with the control). Scale bars, $100 \mu \mathrm{m}$. 


\subsection{Experiment 3: Actin Polymerization Inhibition}

Actin protein is present as either a free monomer called G-actin or as part of a linear polymer microfilament called F-actin, both of which are essential for cellular movement and adhesion during cell division. The actin polymerization inhibitor, cytochalasin, causes a decrease in F-actin and an increase in G-actin content [21]. It has previously been reported that cytochalasin inhibits blastomere adhesion at the cleavage and morula stages [22,23]. To examine whether the inhibition of actin polymerization had an effect on blastomere fusion, cytochalasin $\mathrm{B}(\mathrm{CB})$ was applied to 2-cell EP embryos before and/or after EP (Figure 4A). In brief, $C B$ treatment was conducted for 20 min before $E P(C B+/ C B-), 20$ min after EP $(\mathrm{CB}-/ \mathrm{CB}+)$, or $20 \mathrm{~min}$ before and after $\mathrm{EP}(\mathrm{CB}+/ \mathrm{CB}+)$. The embryos treated with $\mathrm{CB}$ demonstrated no apparent morphological change compared with control embryos (Figure 4B). However, $\mathrm{CB}$ treatment significantly inhibited blastomere fusion following EP (Figure $4 \mathrm{C}, \mathrm{D}$ ). The fusion rate of blastomeres was $23 \%$ in the control and $25 \%$ in $\mathrm{CB}-/ \mathrm{CB}+$ embryos, compared with $10 \%$ in $\mathrm{CB}+/ \mathrm{CB}-$ and $3 \%$ in $\mathrm{CB}+/ \mathrm{CB}+$ embryos. This result inidicates that $\mathrm{CB}$ treatment spanning $\mathrm{EP}(\mathrm{CB}+/ \mathrm{CB}+)$ is the most effective to inhibit blastomere fusion. In contrast, we expected the combined effect of $\mathrm{Ca}^{2+}$-free and $\mathrm{CB}$ treatment on blastomere fusion, but this did not have a better effect than the independent treatment of $\mathrm{Ca}^{2+}$-free or CB (Figure S3).

To investigate the effects of $\mathrm{CB}$ treatment on flox efficiency, sequential EP was performed. The first $\mathrm{EP}$ was performed at the 1-cell stage under standard $\mathrm{CB}$ absent $(\mathrm{CB}-)$ conditions; the second $\mathrm{EP}$ at the 2-cell stage was performed under $\mathrm{CB}+/ \mathrm{CB}-, \mathrm{CB}+/ \mathrm{CB}+$, or control $(\mathrm{CB}-/ \mathrm{CB}-)$ conditions. It was observed that $\mathrm{CB}$ treatment significantly inhibited cell fusion at the 2-cell stage, particularly in the $\mathrm{CB}+/ \mathrm{CB}+$ condition (Figure 4E). Importantly, $\mathrm{CB}$ treatment did not have negative effects on blastocyst development and KI efficiency after sequential EP (Figure 4E). To examine the in vivo development of $\mathrm{CB}$ - treated embryos, floxed mice were generated by $\mathrm{CB}+/ \mathrm{CB}+$ treatment or control $(\mathrm{CB}-/ \mathrm{CB}-)$. Confirming the in vitro results, blastomere fusion of the 2-cell stage embryo was significantly inhibited in the $\mathrm{CB}+/ \mathrm{CB}+$ condition compared with the control (Figure $5 \mathrm{~A}, \mathrm{~B}$ ). $\mathrm{CB}$ treatment did not have negative effects on in vivo development, KI or flox efficiency (Figure 5A,B). Furthermore, the optimized method was successful in both B6D2F1 and B6 mouse strains. These data suggest that actin polymerization inhibition can successfully reduce the rate of blastomere fusion in 2-cell EP embryos without any negative effects. 
A

\begin{tabular}{|c|c|c|c|c|c|c|}
\hline & & $20 \mathrm{mi}$ & & $20 \mathrm{mir}$ & $60 \mathrm{~min}$ & $\begin{array}{l}\text { rusion } \\
\text { check }\end{array}$ \\
\hline CB+/CB- & CB- & $\mathrm{CB}+$ & EP & \multicolumn{2}{|c|}{ CB- } & \\
\hline $\mathrm{CB}+/ \mathrm{CB}+$ & CB- & $\mathrm{CB}+$ & EP & $\mathrm{CB}+$ & CB- & \\
\hline 8- /CB+ & \multicolumn{2}{|c|}{ CB- } & EP & $\mathrm{CB}+$ & CB- & \\
\hline 8- /CB & \multicolumn{2}{|c|}{ CB- } & EP & \multicolumn{2}{|c|}{ CB- } & \\
\hline
\end{tabular}

B
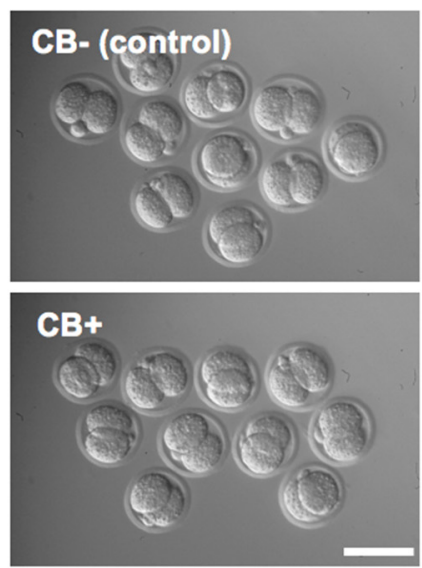

D

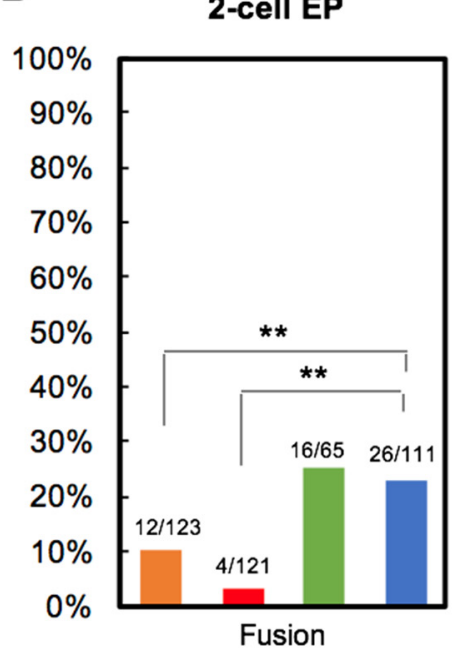

C
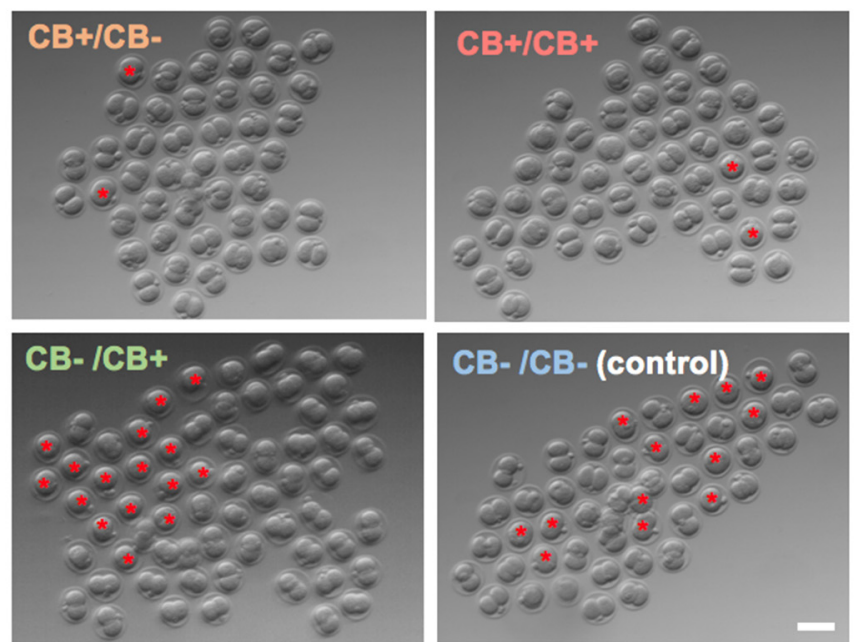

E

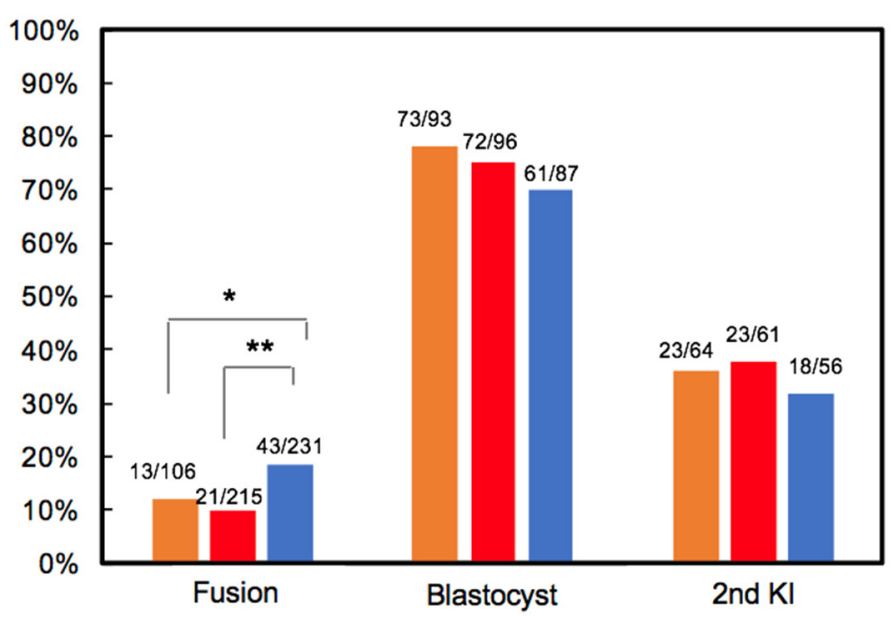

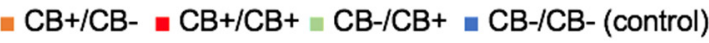

Figure 4. Effects of $\mathrm{CB}$ treatment on blastomere fusion, embryonic development and KI efficiency in vitro. (A) Experimental timeline of CB treatment. (B) Morphology of 2-cell stage embryos (B6D2F1) after 20 min treatment of CB $(5 \mu \mathrm{g} / \mathrm{mL})$. (C) Morphology of 2-cell EP embryos. Asterisks indicate fused embryos. (D) Fusion rate of 2-cell EP embryos. (E) Fusion rate, development (blastocyst) rate, and KI efficiency after sequential EP. * $p<0.05,{ }^{* *} p<0.01$. Scale bars, $100 \mu \mathrm{m}$. 

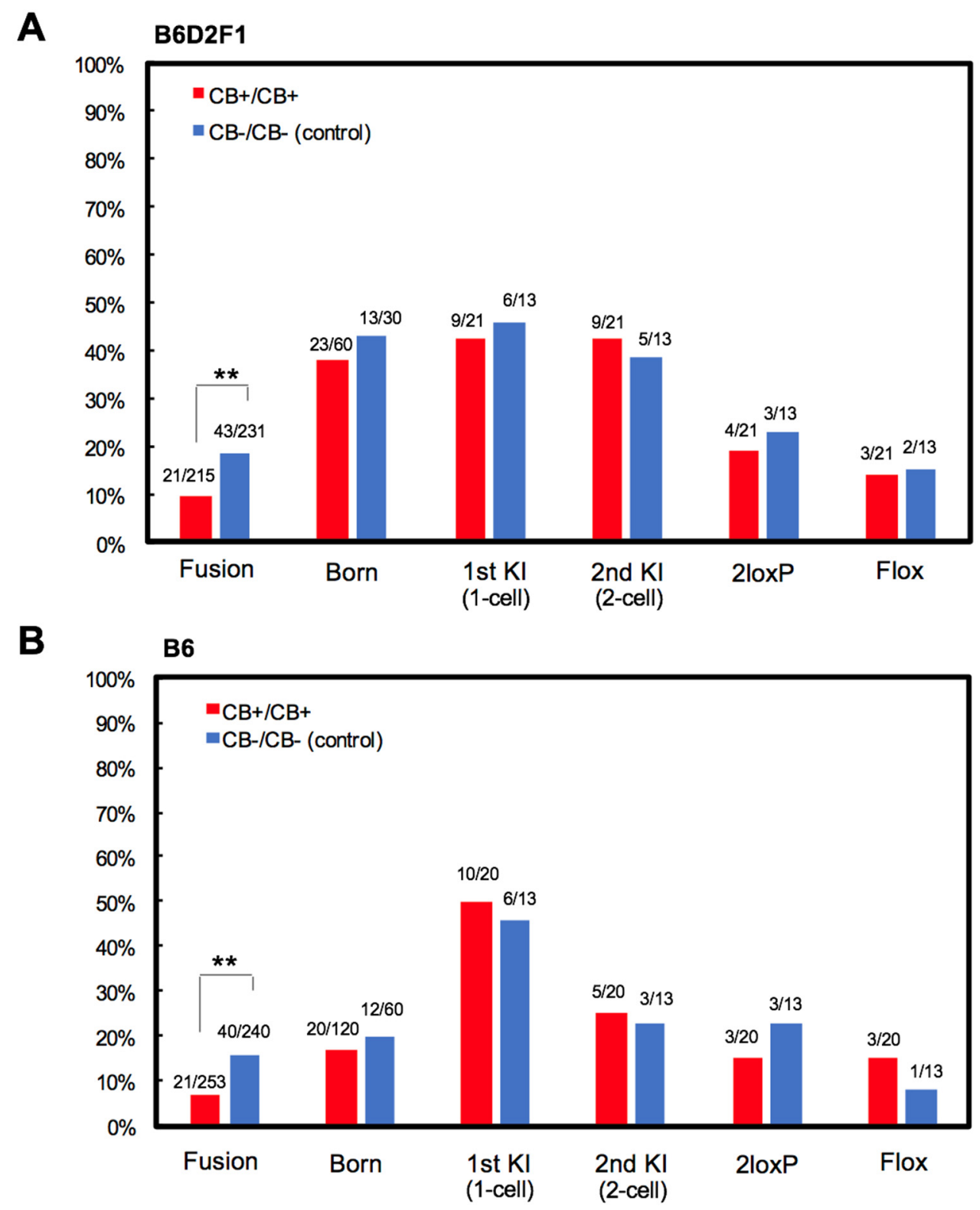

Figure 5. Effects of $\mathrm{CB}$ treatment on 2-cell embryos treated with sequential EP in vivo. Fusion rate, birth rate (born/ET), and KI efficiency after sequential EP were measured in both (A) B6D2F1 and (B) B6 mice. 2loxP $=$ mice with two loxP sites in cis or trans; flox $=$ mice with two loxP sites in cis. ${ }^{* *} p<0.01$.

\section{Discussion}

In this study, the following three conditions were examined to inhibit blastomere fusion by $\mathrm{EP}$ at the 2-cell embryonic stage: (1) hypertonic treatment, (2) $\mathrm{Ca}^{2+}$-free treatment and (3) actin polymerization inhibition.

In Experiment 1, hypertonic treatment of 2-cell stage embryos inhibited blastomere fusion due to repressed cell adherence or the degraded fusogenic state of the cell membrane. The hypertonic condition improved blastocyst development and reduced KI efficiency, probably via a reduction in blastomere volume caused by water moving out of the cell to compensate for increased osmotic pressure. Therefore, the total uptake of Cas9/crRNA/tracrRNA/ssODN would decrease due to reduced blastomere volume. Furthermore, ssODN is toxic to the embryo; therefore, the concentration of ssODN delivered by EP would directly affect embryonic development rate [10]. Thus, when the ssODN uptake is decreased under hypertonic conditions, it is expected that the developmental rate will increase and 
KI efficiency will decrease. Alternatively, cell membrane permeability was potentially degraded by hypertonic treatment, leading to a decrease in Cas9/crRNA/tracrRNA/ssODN uptake following EP. In support of this hypothesis, propidium iodide (PI) uptake by EP in B16-F1 and CHO cell lines was higher in the hypotonic versus the isotonic buffer [15]. Otherwise, EP, especially sequential EP, under isotonic conditions may damage the embryos. Hypertonic treatment before and after EP will reduce embryo damage but lead to a reduced KI efficiency.

$\mathrm{Ca}^{2+}$-free treatment is an established method to reduce cell adhesion. In Experiment 2, PBS(-) treatment partially inhibited cell fusion; however, 2-cell stage embryos had reduced viability and development. PBS(-) treatment after EP was detrimental to embryo viability and may be toxic to cell membrane recovery from electric damage. By contrast, $\mathrm{Ca}^{2+}$-free $\mathrm{M} 2$ treatment also inhibited cell fusion and did not have negative effects on in vitro development and KI rate. To efficiently inhibit cell fusion, a combination of $\mathrm{Ca}^{2+}$-free buffer and another cell adherence inhibitor, such as Ethylenediaminetetraacetic acid (EDTA), known as $\mathrm{Ca}^{2+}$ chelating agent or Cadherin inhibitor(s), may be effective and could be investigated further.

In Experiment 3, blastomere fusion rate was investigated after actin polymerization inhibition. Embryos at the cleavage-stage have an abundance of actin immediately beneath the cell membrane, which is occasionally located inter-cellularly $[24,25]$. Previous studies indicate that the actin polymerization inhibitor, cytochalasin, can prevent blastomere adhesion in preimplantation embryos. De-compaction of morula-stage embryos can be induced by treatment with cytochalasin [23]. Furthermore, 2-cell stage embryos treated with cytochalasin for $44-48$ h have well-separated blastomeres, indicating a loss of adhesion [22]. Long-term treatment with cytochalasin usually induces polyploidy because cytochalasin allows chromosomal replication to proceed while arresting cytoplasmic division. Therefore, short-time treatment $(20 \mathrm{~min})$ of $\mathrm{CB}$ was tested in this experiment. Despite no observable morphological changes, cell fusion was significantly inhibited by $\mathrm{CB}$ treatment before and after EP. Further, CB treatment had no effect on either in vitro or in vivo development, nor on KI efficiency. The blastomere fusion cannot be completely suppressed by CB treatment; therefore, we expected the combined effect of $\mathrm{Ca}^{2+}$-free and $\mathrm{CB}$ treatment on blastomere fusion. However, this did not have a better effect than the independent treatment of $\mathrm{Ca}^{2+}$-free or $\mathrm{CB}$ treatment. $\mathrm{CB}$ treatment time or other actin polymerization inhibitors (e.g., Latrunculin A) can be further optimized and warrant further testing. Theoretically, CB treatment could achieve a $10 \%$ increase in floxed mice as a result of a $10 \%$ reduction in blastomere fusion. Together, these data suggest that $\mathrm{Ca}^{2+}$-free treatment and the inhibition of actin polymerization at the 2-cell stage can generate floxed mice by a sequential EP method.

In recent years, various methods have been reported as potential alternatives to the two-donor floxing technique. Methods using long single-stranded DNA (lssDNA) as donors are known as Easi-CRISPR (efficient additions with ssDNA inserts-CRISPR) [26], or CLICK (CRISPR with lssDNA inducing cKO alleles) [27]. Both techniques introduce lssDNA as follows: Easi-CRISPR is a microinjection-based approach, whereas CLICK is an EP-based approach. Alternatively, Tild-CRISPR (targeted integration with linearized dsDNA-CRISPR) uses long-dsDNA as donors [28]. These newer techniques achieve an improved KI efficiency compared with the simultaneous introduction of two pairs of ssODNs and gRNAs [11]. Unlike the two-donor floxing technique, the new methods are limited by the lengths of the homology arms and of the loxP flanked region. The sequential KI method improved the flox rate compared with simultaneous KI. Using sequential EP, floxed mice were successfully generated with more than $20 \mathrm{~kb}$ of flanking region by two loxP sites (data not shown). Recently, a new approach was reported called sequential $i$-GONAD (si-GONAD) [29], which sequentially electroporates two gRNAs (and two ssODNs) via oviductal nucleic acid delivery. Although the sequential EP method requires further improvement for not only the inhibition of blastomere fusion at 2-cell stage, but also improvement of embryonic development and successful KI, it has great potential alongside other floxing methods. 


\section{Conclusions}

Sequential EP of loxP donors in 1- or 2-cell stage embryos is more efficient than simultaneous EP for the generation of floxed mice. However, a limitation of EP at the 2-cell stage is the frequency of blastomere fusion, which is embryonic lethal because of tetraploidy. Optimization of the sequential EP method revealed that $\mathrm{Ca}^{2+}$-free treatment and actin polymerization inhibition effectively reduced fusion rate, thereby increasing the number of viable embryos to obtain more floxed mice.

Supplementary Materials: The following are available online at http://www.mdpi.com/2073-4409/9/5/1088/s1. Figure S1: The morphology of embryos by hypertonic treatment. Figure S2: Effects of PBS(-) treatment on blastomere fusion and embryonic development of 2-cell EP. Figure S3: The combined $\mathrm{Ca}^{2+}$-free and CB treatment does not have a better effect on blastomere fusion than the only $\mathrm{Ca}^{2+}$-free treatment.

Author Contributions: Conceptualization, T.H. and I.H.; investigation, T.H., R.K., M.K. and S.M.; writing-original draft preparation, T.H.; writing - review and editing, I.H. All authors have read and agreed to the published version of the manuscript.

Funding: This work was supported by the Basic Science and Platform Technology Program for Innovative Biological Medicine from the Ministry of Education, Culture, Sports, Science and Technology, Japan (MEXT) to T.H., S.M., and I.H.; the Japan Agency for Medical Research and Development (AMED) to I.H.; Platform Project for Supporting Drug Discovery and Life Science Research (Basis for Supporting Innovative Drug Discovery and Life Science Research (BINDS)) from AMED under Grant Number JP19am0101120 to I.H.; the Tokyo Biochemical Research Foundation to I.H.; and the Takeda Science Foundation to I.H.

Acknowledgments: We thank Eriko Suetomo for technical support of the assay for KI and floxed allele.

Conflicts of Interest: The authors declare no conflict of interest.

\section{References}

1. Dickinson, M.E.; Flenniken, A.M.; Ji, X.; Teboul, L.; Wong, M.D.; White, J.K.; Meehan, T.F.; Weninger, W.J.; Westerberg, H.; Adissu, H.; et al. High-throughput discovery of novel developmental phenotypes. Nature 2016, 537, 508-514. [CrossRef]

2. Sauer, B.; Henderson, N. Site-specific DNA recombination in mammalian cells by the Cre recombinase of bacteriophage P1. Proc. Natl. Acad. Sci. USA 1988, 85, 5166-5170. [CrossRef] [PubMed]

3. Jinek, M.; Chylinski, K.; Fonfara, I.; Hauer, M.; Doudna, J.A.; Charpentier, E. A programmable dual-RNAguided DNA endonuclease in adaptive bacterial immunity. Science 2012, 337, 816-821. [CrossRef] [PubMed]

4. Wang, H.; Yang, H.; Shivalila, C.S.; Dawlaty, M.M.; Cheng, A.W.; Zhang, F.; Jaenisch, R. One-step generation of mice carrying mutations in multiple genes by CRISPR/Cas-mediated genome engineering. Cell 2013, 153, 910-918. [CrossRef] [PubMed]

5. Ran, F.A.; Hsu, P.D.; Lin, C.Y.; Gootenberg, J.S.; Konermann, S.; Trevino, A.E.; Scott, D.A.; Inoue, A.; Matoba, S.; Zhang, Y.; et al. Double nicking by RNA-guided CRISPR Cas9 for enhanced genome editing specificity. Cell 2013, 154, 1380-1389. [CrossRef] [PubMed]

6. Yang, H.; Wang, H.; Shivalila, C.S.; Cheng, A.W.; Shi, L.; Jaenisch, R. One-step generation of mice carrying reporter and conditional alleles by CRISPR/Cas-mediated genome engineering. Cell 2013, 154, 1370-1379. [CrossRef] [PubMed]

7. Bishop, K.A.; Harrington, A.; Kouranova, E.; Weinstein, E.J.; Rosen, C.J.; Cui, X.; Liaw, L. CRISPR/Cas9Mediated Insertion of loxP Sites in the Mouse Dock7 Gene Provides an Effective Alternative to Use of Targeted Embryonic Stem Cells. G3 2016, 6, 2051-2061. [CrossRef]

8. Nakagawa, Y.; Oikawa, F.; Mizuno, S.; Ohno, H.; Yagishita, Y.; Satoh, A.; Osaki, Y.; Takei, K.; Kikuchi, T.; Han, S.I.; et al. Hyperlipidemia and hepatitis in liver-specific CREB3L3 knockout mice generated using a one-step CRISPR/Cas9 system. Sci. Rep. 2016, 6, 27857. [CrossRef]

9. Ma, X.; Chen, C.; Veevers, J.; Zhou, X.; Ross, R.S.; Feng, W.; Chen, J. CRISPR/Cas9-mediated gene manipulation to create single-amino-acid-substituted and floxed mice with a cloning-free method. Sci. Rep. 2017, 7, 42244. [CrossRef]

10. Horii, T.; Morita, S.; Kimura, M.; Terawaki, N.; Shibutani, M.; Hatada, I. Efficient generation of conditional knockout mice via sequential introduction of lox sites. Sci. Rep. 2017, 7, 7891. [CrossRef] 
11. Gurumurthy, C.B.; O’Brien, A.R.; Quadros, R.M.; Adams, J., Jr.; Alcaide, P.; Ayabe, S.; Ballard, J.; Batra, S.K.; Beauchamp, M.C.; Becker, K.A.; et al. Reproducibility of CRISPR-Cas9 methods for generation of conditional mouse alleles: A multi-center evaluation. Genome Biol. 2019, 20, 171. [CrossRef] [PubMed]

12. Horii, T.; Yamamoto, M.; Morita, S.; Kimura, M.; Nagao, Y.; Hatada, I. p53 suppresses tetraploid development in mice. Sci. Rep. 2015, 5, 8907. [CrossRef]

13. Hogan, B.; Constantini, F.; Lacy, E. Manipulating the Mouse Embryo: A Laboratory Manual, 1st ed.; Cold Spring Harbor Laboratory: Huntington, NY, USA, 1986; pp. 252-253.

14. Schmitt, J.J.; Zimmermann, U. Enhanced hybridoma production by electrofusion in strongly hypo-osmolar solutions. Biochim. Biophys. Acta 1989, 983, 42-50. [CrossRef]

15. Usaj, M.; Kanduser, M. The systematic study of the electroporation and electrofusion of B16-F1 and CHO cells in isotonic and hypotonic buffer. J. Membr. Biol. 2012, 245, 583-590. [CrossRef] [PubMed]

16. Takeichi, M. Cadherin cell adhesion receptors as a morphogenetic regulator. Science 1991, 251, 1451-1455. [CrossRef] [PubMed]

17. Larue, L.; Ohsugi, M.; Hirchenhain, J.; Kemler, R. E-cadherin null mutant embryos fail to form a trophectoderm epithelium. Proc. Natl. Acad. Sci. USA 1994, 91, 8263-8267. [CrossRef]

18. Riethmacher, D.; Brinkmann, V.; Birchmeier, C. A targeted mutation in the mouse E-cadherin gene results in defective preimplantation development. Proc. Natl. Acad. Sci. USA 1995, 92, 855-859. [CrossRef]

19. De Vries, W.N.; Evsikov, A.V.; Haac, B.E.; Fancher, K.S.; Holbrook, A.E.; Kemler, R.; Solter, D.; Knowles, B.B. Maternal beta-catenin and E-cadherin in mouse development. Development 2004, 131, 4435-4445. [CrossRef]

20. Johnson, M.H.; Maro, B.; Takeichi, M. The role of cell adhesion in the synchronization and orientation of polarization in 8-cell mouse blastomeres. J. Embryol. Exp. Morphol. 1986, 93, 239-255.

21. Cooper, J.A. Effects of cytochalasin and phalloidin on actin. J. Cell Biol. 1987, 105, 1473-1478. [CrossRef]

22. Kimber, S.J.; Surani, M.A. Morphogenetic analysis of changing cell associations following release of 2-cell and 4-cell mouse embryos from cleavage arrest. J. Embryol. Exp. Morphol. 1981, 61, 331-345. [PubMed]

23. Pey, R.; Vial, C.; Schatten, G.; Hafner, M. Increase of intracellular $\mathrm{Ca}^{2+}$ and relocation of E-cadherin during experimental decompaction of mouse embryos. Proc. Natl. Acad. Sci. USA 1998, 95, 12977-12982. [CrossRef] [PubMed]

24. Lehtonen, E.; Badley, R.A. Localization of cytoskeletal proteins in preimplantation mouse embryos. J. Embryol. Exp. Morphol. 1980, 55, 211-225.

25. Duan, X.; Zhang, H.L.; Wu, L.L.; Liu, M.Y.; Pan, M.H.; Ou, X.H.; Sun, S.C. Involvement of LIMK1/2 in actin assembly during mouse embryo development. Cell Cycle 2018, 17, 1381-1389. [CrossRef]

26. Quadros, R.M.; Miura, H.; Harms, D.W.; Akatsuka, H.; Sato, T.; Aida, T.; Redder, R.; Richardson, G.P.; Inagaki, Y.; Sakai, D.; et al. Easi-CRISPR: A robust method for one-step generation of mice carrying conditional and insertion alleles using long ssDNA donors and CRISPR ribonucleoproteins. Genome Biol. 2017, 18, 92. [CrossRef]

27. Miyasaka, Y.; Uno, Y.; Yoshimi, K.; Kunihiro, Y.; Yoshimura, T.; Tanaka, T.; Ishikubo, H.; Hiraoka, Y.; Takemoto, N.; Tanaka, T.; et al. CLICK: One-step generation of conditional knockout mice. BMC Genom. 2018, 19, 318. [CrossRef]

28. Yao, X.; Zhang, M.; Wang, X.; Ying, W.; Hu, X.; Dai, P.; Meng, F.; Shi, L.; Sun, Y.; Yao, N.; et al. Tild-CRISPR Allows for Efficient and Precise Gene Knockin in Mouse and Human Cells. Dev. Cell 2018, 45, 526-536. [CrossRef]

29. Sato, M.; Miyagasako, R.; Takabayashi, S.; Ohtsuka, M.; Hatada, I.; Horii, T. Sequential i-GONAD: An Improved In Vivo Technique for CRISPR/Cas9-Based Genetic Manipulations in Mice. Cells 2020, 9, 546. [CrossRef]

(C) 2020 by the authors. Licensee MDPI, Basel, Switzerland. This article is an open access article distributed under the terms and conditions of the Creative Commons Attribution (CC BY) license (http://creativecommons.org/licenses/by/4.0/). 